\title{
Low triiodothyronine syndrome: a prognostic marker for outcome in sepsis?
}

\author{
Stefanie Meyer $\cdot$ Philipp Schuetz $\cdot$ Melanie Wieland • \\ Charly Nusbaumer $\cdot$ Beat Mueller . \\ Mirjam Christ-Crain
}

Revised: 2 November 2010/ Accepted: 11 November 2010/Published online: 6 January 2011

(C) Springer Science+Business Media, LLC 2011

\begin{abstract}
There is ongoing controversy as to whether hormonal changes of the euthyroid sick syndrome are predictors of poor outcome in sepsis and critical illness. In this prospective study, the prognostic accuracy of thyroid hormone levels in 103 critically ill adult patients on admission and during follow up in a medical intensive care unit (ICU) was assessed and was compared to clinical risk scores, namely, the acute physiology and chronic health evaluation and the simplified acute physiology score. Median T3 levels on admission to the ICU were lower in the 53 septic cases [0.9 $\mathrm{nmol} / \mathrm{l}$ (IQR 0.6-1.1)] as compared with the 50 patients with a systemic inflammatory response syndrome $[1.2 \mathrm{nmol} /$ 1 (IQR 0.8-1.4), $P<0.001]$. The lowest T3 levels were found in patients with severe sepsis $[0.8 \mathrm{nmol} / \mathrm{l}$ (IQR 0.55-0.95)] and septic shock [0.8 nmol/l (IQR 0.6-1.0)]. There was no difference in T3 and free thyroxin (fT4) levels on admission in non-survivors compared with survivors overall and in subgroups of patients with SIRS and sepsis. During the follow up, fT4 levels decreased significantly in non-survivors, while they increased in survivors [fT4
\end{abstract}

Stefanie Meyer and Philipp Schuetz contributed equally to this study.

S. Meyer $\cdot$ P. Schuetz $\cdot$ M. Wieland $\cdot$ M. Christ-Crain Department of Internal Medicine, Division of Endocrinology Diabetes and Clinical Nutrition, University Hospital Basel, Basel, Switzerland

e-mail: meyerste@uhbs.ch

C. Nusbaumer

Department of Chemical Pathology,

University Hospital Basel, Basel, Switzerland

B. Mueller $(\bowtie)$

Department of Internal Medicine, Kantonsspital Aarau,

Tellstrasse, 5001 Aarau, Switzerland

e-mail: Happy.Mueller@unibas.ch difference -1.3 (IQR -2.5 to 0.2 ) vs. 0.8 (IQR -0.85 to 4.1 ), $P=0.003]$. In addition, on the day of death, non-survivors had lower T3 and fT4 levels as compared with survivors $(P=0.04$ and $P=0.02)$. T3 and fT4 levels on admission were not prognostic in this cohort of critically ill patients. A decrease in fT4 levels in the course of disease, however, may point to adverse outcome.

Keywords Sepsis - Triiodothyronine - Free thyroxin . Outcome

\section{Introduction}

Sepsis affects 750,000 people annually and accounts for 210,000 deaths every year in the USA despite improved medical treatment strategies [1,2]. To tackle the challenges of early diagnosis and risk stratification, several clinical prognostic scores have been developed. For this purpose, the updated Acute Physiology and Chronic Health Evaluation II (APACHE) and the Simplified Acute Physiology Score II (SAPS) are extensively validated prognostic assessment tools used in critically ill patients for stratification of disease severity and estimation of in-hospital mortality based on routine physiologic measurements, age, and clinical parameters [3, 4]. Because the prognostic accuracy of these clinical scores still demonstrates a level of uncertainty, there is interest for novel prognostic biomarkers, each mirroring different pathophysiological mechanisms. In this context, endocrine markers such as plasma concentrations of cortisol, adrenomedullin, and growth hormone have been reported to correlate with severity and outcome of septic adults and children [5-8]. Severity-dependent alterations in the thyroid axis during critical illness are well known. The constellation of low 
total triiodothyronine (T3) levels, increased reverse T3 levels, and/or low total thyroxin (T4) levels with normal free thyroxin (fT4) levels in the absence of an obvious thyroid disease is commonly called the "euthyroid sick syndrome" [9-11]. Some studies from different clinical settings suggested that low levels of thyroid hormones are predictors of poor outcome in sepsis and critical illness [12-15]. However, other studies found no, or even opposite, associations of thyroid hormone concentrations with outcome [16, 17]. Thus, there still remains a controversy as to whether changes in thyroid hormone levels add prognostic information to the widely used clinical risk scores. We therefore assessed the prognostic accuracy of thyroid hormone levels in a cohort of critically ill adult patients on admission and during follow up in a medical intensive care unit (ICU).

\section{Materials and methods}

In this prospective study, thyroid hormone values in plasma samples of 103 critically ill patients in the medical ICU of the University Hospital of Basel, Switzerland were evaluated. The primary objective of this study was to evaluate the prognostic value of endocrine dysfunction in critical illness (PEDCRIP study) [18]. More specifically, the study aimed to evaluate the prognostic accuracy of thyroid- and other hypothalamus-pituitary-axis hormones to predict death in the ICU setting $[18,19]$. A total of 103 critically ill patients were enrolled over a 9-month period. Assessment of patients on admission was performed three times, i.e., during the first $24 \mathrm{~h}$, on day 2, and at discharge from the ICU or death. Clinical status, vital signs, and severity of disease were monitored daily including the calculation of the APACHE II and SAPS II. Depending on the clinical status, informed consent was obtained from the patient or its relatives. Prior approval of the study protocol was obtained from the ethical committee.

Patients were classified into systemic inflammatory response syndrome (SIRS) without underlying infection and sepsis including severe sepsis and septic shock based on international consensus criteria [20, 21]. Classification of patients was done after discharge of patients on the basis of patient-chart review, results of microbiological cultures, radiographs, and if available by post-mortem examination reports. In case of uncertainty, an infectious disease specialist was asked for additional review of the case. An isolated microorganism was considered to be pathogenic if detected within $24 \mathrm{~h}$ before or after the onset of systemic response. Colonization with bacteria (e.g., bacteruria in an asymptomatic patient with a urine catheter) or post-mortem positive blood cultures were disregarded.

\section{Assay}

Blood samples were obtained from an indwelling venous or arterial catheter. Routine blood count, C-reactive protein (CRP), procalcitonin, and interleukin 6 (IL6) levels were measured in all the patients. CRP (mg/l) was measured by an enzyme immunoassay (EMIT C-reactive protein assay; E. Merck Diagnostica Zurich, Switzerland). Procalcitonin (PCT) (ng/ml) was measured using the immunoluminometric assay (LUMItest ${ }^{\circledR}$ PCT; Brahms Diagnostica Berlin, Germany) with a lower detection limit of $0.1 \mu \mathrm{g} / \mathrm{l}$. Serum IL6 concentrations $(\mathrm{ng} / \mathrm{ml})$ were measured with a commercially available quantitative sandwich enzyme immunoassay (CLB; Pelikine CompactTM, Amsterdam, Netherlands) with a detection limit of $0.6 \mathrm{pg} / \mathrm{ml}$.

Plasma was collected at the time of blood sampling on admission, day 2 and at discharge from the ICU or death, respectively, in plastic tubes containing ethylenendiaminetatraacetic (EDTA). The tubes were placed on ice, centrifuged at 3,000 $\mathrm{g}$, and plasma was stored at $-70^{\circ} \mathrm{C}$ until assayed. T3 (nmol/l) and fT4 (pmol/l) concentrations were measured by an electro-chemistry-luminescence immunoassay (ECLIA, Roche Diagnostics, Mannheim, Germany) with a functional detection range of $0.3-10 \mathrm{nmol} / \mathrm{l}$ and 0.3-100 pmol/l, respectively.

We preferred measurement of total T3 over free T3 because in our experience and also according to the literature, free T3 measurement shows greater variability, and thus the precision is less reliable for use in clinical practice [22].

\section{Statistical analysis}

All data are presented as medians and interquartile ranges. Levels that were not detectable were considered to have a value equal to the lower limit of detection of the assay. Frequency comparison was done by chi-square test. For not normally distributed two-group comparison, the MannWhitney- $U$ test was used, and the Kruskal-Wallis one-way analysis of variance was used if more than two groups were being compared. Correlation analyses were performed using Spearman rank correlation. These tests were done using Graph Pad Prism (version 4, GraphPAd, la Jolla, CA, USA) and STATISTICA (version 6, StatSoft, Tulsa, OK, USA). Receiver-operating-characteristics (ROCs) were calculated using MedCalc for windows (version 7.2.1.0., Mariakerke, Belgium).

All the statistic tests were two-tailed, and $P$ values less than 0.05 were considered to indicate statistical significance. 


\section{Results}

Descriptive characteristics of patients

The median age of the 103 patients included in this study was 59 years (IQR 46-68.5) with 54.4\% males. On admission, the median APACHE II was 21 points (IQR 17-27.5) and the median SAPS II was 54 points (IQR 43.5-66). The median length of stay in the medical ICU was 3 days (IQR 1-4). On admission, SIRS without underlying infection was diagnosed in 50 patients and sepsis in 53 patients including 15 patients with severe sepsis and 16 patients with septic shock. Patient's underlying diagnoses are summarized in Table 1.

Table 1 Clinical diagnoses of patients

\begin{tabular}{|c|c|}
\hline Clinical diagnoses of patients & $\begin{array}{l}\text { Number of } \\
\text { patients }\end{array}$ \\
\hline \multicolumn{2}{|l|}{ Respiratory } \\
\hline Pneumonia & 33 \\
\hline Chronic obstructive pulmonary disease & 14 \\
\hline Asthma & 3 \\
\hline Pharyngeal obstruction & 2 \\
\hline Toxic pulmonary edema & 2 \\
\hline Bronchiale carcinoma & 3 \\
\hline \multicolumn{2}{|l|}{ Intestinal } \\
\hline Abdominal infection & 6 \\
\hline Urinary tract infection & 5 \\
\hline Gastrointestinal bleeding & 7 \\
\hline Intestinal ischemia & 1 \\
\hline Renal failure & 1 \\
\hline Hepatic coma & 3 \\
\hline \multicolumn{2}{|l|}{ Cardiovascular } \\
\hline Myocardial infarction & 12 \\
\hline Heart failure & 11 \\
\hline Pulmonary embolism & 4 \\
\hline Hemorrhagic shock & 1 \\
\hline \multicolumn{2}{|l|}{ Cerebral } \\
\hline Intracranial hemorrhage & 3 \\
\hline Ischemic stroke & 5 \\
\hline Subarachnoidal hemorrhage & 4 \\
\hline Meningitis & 1 \\
\hline Epilepsy & 3 \\
\hline Sinus venous thrombosis & 1 \\
\hline \multicolumn{2}{|l|}{ Other diagnosis } \\
\hline Intoxication & 4 \\
\hline Diabetic coma & 3 \\
\hline Postoperative & 4 \\
\hline Others & 2 \\
\hline Leukemia & 7 \\
\hline
\end{tabular}

One patient can have more than one diagnosis, therefore the total exceeds the absolute number of patients
The principal site of infection was the lung $(n=37)$, the gastrointestinal tract $(n=5)$, the urogenital tract $(n=4)$, and blood stream infection $(n=2)$. Blood cultures showed growth of typical microorganisms in 31 patients including Streptococcus pneumoniae $(n=9), \quad$ Pseudomonas aeruginosa $(n=6)$, E. coli $(n=6)$, Enterobacteriaceae $(n=2)$, Haemophilus influenzae $(n=2)$, and Staphylococcus aureus $(n=1) .30 \%$ of patients with SIRS were treated with dopamine for blood pressure support compared to $49.1 \%$ of patients with sepsis. IL6 levels were significantly higher in patients with sepsis compared to patients with SIRS $(P<0.0001)$. Mortality rates of patients with and without infection were similar: $22 \%$ of patients with SIRS and $24.5 \%$ of patients with sepsis died in the hospital. Baseline data of the whole patient cohort and those grouped into survivors and non-survivors are summarized in Table 2. No statistically significant difference was detected between survivors and non-survivors concerning age, sex, and common clinical parameters.

Prognostic assessment on admission showed a significantly higher APACHE II and SAPS II in non-survivors as compared with survivors [27 (IQR 19-31.8) vs. 21 (IQR 16-25.5) and 67 (IQR 53.8-83) vs. 50 (IQR 39-62)]. Similarly, IL6 was higher in non-survivors compared with survivors $[260 \mathrm{ng} / \mathrm{ml}$ (IQR 64.9-1167.5) vs. $48.9 \mathrm{ng} / \mathrm{ml}$ (IQR 9.3-177.5)].

Thyroid hormones on admission and during follow-up

The median T3 level of the 103 patients on admission was $1.0 \mathrm{nmol} / \mathrm{l}$ (IQR 0.7-1.2). In 66 patients (65\%), a T3 level below the lower reference range of $1.2 \mathrm{nmol} / 1$ was found, while the other 36 patients $(35 \%)$ had a T3 within the normal range. The median fT4 level was $14.2 \mathrm{pmol} / \mathrm{l}$ (IQR 11.6-16.6). Twenty-five patients (24\%) had fT4 levels below, 71 patients $(69 \%)$ within and 6 patients $(6 \%)$ above the normal reference range of 11.6-22.0 pmol/1.

On day $2,58 \%$ of patients had T3 levels and $23 \%$ of patients had fT4 levels below the reference range. On the day of discharge or death, respectively, T3 levels and fT4 levels below the reference range were found in $57 \%$ and $26 \%$ of patients.

T3 levels on admission correlated with serum PCT levels $(r=0.44, P<0.001)$ but not with the APACHE II $(r=-0.14, P=0.14)$ or SAPS II $(r=0.15, P=0.13)$. FT4 levels did not correlate with PCT levels $(r=0.14$, $P=0.16)$, the APACHE II $(r=-0.12, P=0.22)$, or the SAPS II $(r=0.17, P=0.07)$.

T3 and fT4 levels in patients with SIRS, sepsis, severe sepsis, and septic shock

Median T3 levels on admission to the ICU were lower in septic patients [0.9 nmol/1 (IQR 0.6-1.1)] as compared to 
Table 2 Baseline data

\begin{tabular}{|c|c|c|c|c|}
\hline Charcteristics & All patients $(n=103)$ & Survivors $(n=79)$ & Non-survivors $(n=24)$ & $P$ \\
\hline \multicolumn{5}{|l|}{ Demographic characteristics } \\
\hline Age-years-(median-IQR) & $59(46-68)$ & $56(46-67)$ & $63(52.5-73.3)$ & 0.1 \\
\hline Female sex $(\%)$ & $46(n=47)$ & $47(n=37)$ & $42(n=10)$ & 0.4 \\
\hline Weight (kg)-median-IQR) & $70.1(62.5-81.3)$ & $70.1(61-84)$ & $71(65-79.3)$ & 0.8 \\
\hline BMI $\left(\mathrm{kg} / \mathrm{m}^{2}\right)$-(median-IQR) & $24.2(22.1-27.3)$ & $24.2(22.2-27.3)$ & $24.2(22.1-25.5)$ & 0.8 \\
\hline \multicolumn{5}{|l|}{ Clinical findings } \\
\hline Heart rate (bpm)-(median-IQR) & $107(82-125)$ & $110(82.5-124)$ & $97.5(79-126)$ & 0.9 \\
\hline Respirator-rate-(median-IQR) & $16(12-25)$ & $16(12-25)$ & $14(10.8-13.3)$ & 0.3 \\
\hline Temperature $\left({ }^{\circ} \mathrm{C}\right)$-(median-IQR) & $36.7(35.8-37.6)$ & $36.8(36-37.7)$ & $36.5(35.5-37.4)$ & 0.3 \\
\hline \multicolumn{5}{|l|}{ Routine laboratory findings-(median-IQR) } \\
\hline C-reactive protein $(\mathrm{mg} / \mathrm{l})$ & $81(17-200)$ & $74(15.5-189)$ & $84(42.8-246.8)$ & 0.5 \\
\hline Procalcitonin $(\mu \mathrm{g} / \mathrm{l})$ & $1.1(0.3-4.8)$ & $1.1(0.3-4.3)$ & $2.4(0.6-11.1)$ & 0.2 \\
\hline IL6 $(\mathrm{pg} / \mathrm{ml})$ & $69.4(12.6-278)$ & $48.9(9.3-177.5)$ & $260(64.9-1167.5)$ & 0.002 \\
\hline White blood count $\left(\times 10^{9}\right)$ & $11.1(8.2-15.2)$ & $10.3(8.2-13.5)$ & $13.6(8.6-18.2)$ & 0.09 \\
\hline \multicolumn{5}{|l|}{ Thyroid hormones (median-IQR) } \\
\hline $\mathrm{T} 3(\mathrm{nmol} / \mathrm{l})$ on admission & $1.0(0.7-1.2)$ & $1.0(0.7-1.3)$ & $0.9(0.7-1.1)$ & 0.3 \\
\hline fT4 $(\mathrm{pmol} / \mathrm{l})$ on admission & $14.2(11.6-16.6)$ & $14.6(11.7-16.5)$ & $13.6(11-17.3)$ & 0.66 \\
\hline T3 $(\mathrm{nmol} / \mathrm{l})$ on day 2 & $1.0(0.8-1.3)$ & $1.1(0.8-1.3)$ & $0.9(0.8-1.1)$ & 0.08 \\
\hline fT4 $(\mathrm{pmol} / \mathrm{l})$ on day 2 & $14.6(11.0-17.1)$ & $14.7(11.6-17.1)$ & $11.8(9.8-17)$ & 0.2 \\
\hline $\mathrm{T} 3(\mathrm{nmol} / \mathrm{l})$ at death or discharge & $0.9(0.7-1.3)$ & $1.0(0.7-1.3)$ & $0.8(0.7-0.9)$ & 0.04 \\
\hline fT4 $(\mathrm{pmol} / \mathrm{l})$ at death or discharge & $14.8(11.2-17.7)$ & $15.3(12.3-18.3)$ & $11.9(6.9-15.8)$ & 0.02 \\
\hline \multicolumn{5}{|l|}{ Prognostic scores on admission } \\
\hline APACHE II (points)-(median-IQR) & $21(17-28)$ & $21(16-25.5)$ & $27(19-31.8)$ & 0.005 \\
\hline SAPS II (points)-(median-IQR) & $54(12.5-284)$ & $50(39-62)$ & $67(53-83)$ & 0.0003 \\
\hline \multicolumn{5}{|l|}{ Co medication } \\
\hline Dopamin treatment $(\%)$ & $39.8(n=41)$ & $36.7(n=29)$ & $50(n=12)$ & 0.32 \\
\hline
\end{tabular}

$B M I$ body mass index, T3 triiodothyronine, fT4 free thyroxin, IL6 interleukin 6, APACHE II acute physiology and chronic health evaluation, SAPS II simplified acute physiology score

patients with SIRS and no infection [1.2 nmol/l (IQR 0.8-1.4), $P<0.001$ ] (Fig. 1). In septic patients, the lowest $\mathrm{T} 3$ levels were found in subgroups of patients with severe sepsis [0.8 nmol/l (IQR 0.6-1.0)] and septic shock [0.8 nmol/l (IQR 0.6-1.0)] (Fig. 2). There was no difference between the fT4 levels in patients with SIRS and in patients with sepsis, as well as in subgroups of patients with severe sepsis and septic shock.

T3 and fT4 levels and outcome of patients

T3 and fT4 levels on admission were not different between non-survivors and survivors $(P=0.32$ and $P=0.67)$ (Fig. 3). The median T3 level in non-survivors was $0.9 \mathrm{nmol} / \mathrm{l}$ (IQR 0.7-1.1) compared to $1.0 \mathrm{nmol} / \mathrm{l}$ (IQR $0.7-1.3$ ) in survivors. The median fT4 level was $13.6 \mathrm{pmol} / 1$ (IQR 11.0-17.3) in non-survivors and $14.6 \mathrm{pmol} / \mathrm{l}$ (IQR 11.7-16.5) in survivors, respectively. Similarly, T3 and fT4 levels were not different between non-survivors and survivors in subgroups of SIRS and septic patients, respectively.

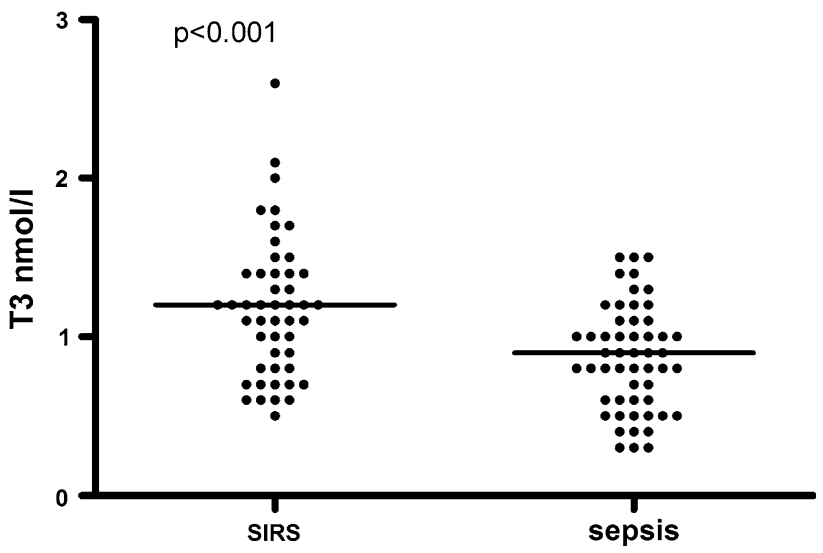

Fig. 1 Admission T3 levels in patients with SIRS and sepsis (including severe sepsis and septic shock). Lines denote median values, each point represents one measurement. T3 triiodothyronine, SIRS systemic inflammatory response syndrome

Overall, there was no statistical difference between T3 $(P=0.08$ and $P=0.46)$ and $\mathrm{fT} 4(P=0.5$ and 0.05$)$ levels on admission as compared to values obtained after 


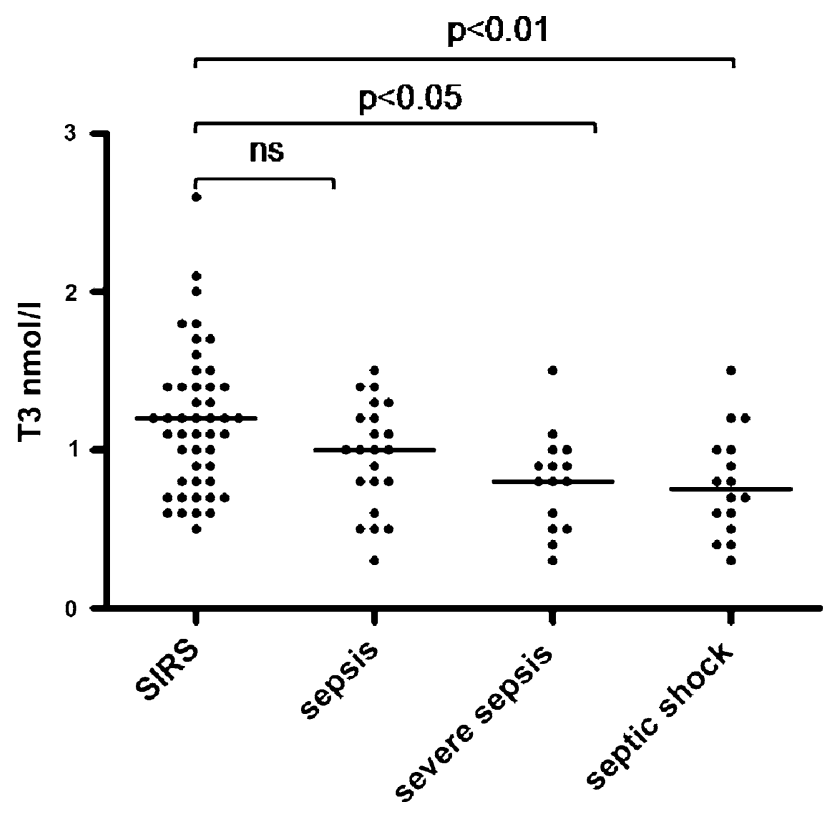

Fig. 2 Admission levels of T3 in patients with SIRS, sepsis, severe sepsis and septic shock. Lines denote median values, each point represents one measurement. T3 triiodothyronine, SIRS systemic inflammatory response syndrome

2 days and at discharge from the ICU. However, during follow up, fT4 levels significantly decreased in nonsurvivors, while an increase in survivors was found [fT4 difference -1.3 (IQR -2.5 to 0.2 ) vs. 0.8 (IQR -0.85 to $4.1), P=0.003]$. On the day of death, non-survivors had lower T3 and fT4 levels as compared with survivors [0.8 nmol/l (IQR 0.7-0.9) vs.1.0 nmol/l (IQR 0.7-1.3), $P=0.04$ and $11.9 \mathrm{pmol} / \mathrm{l}$ (IQR $6.9-15.7) \mathrm{vs} .15 .3 \mathrm{pmol} / \mathrm{l}$ (IQR 12.3-18.3), $P=0.02$ ] (Fig. 4).

To investigate the prognostic potential of T3 and fT4 levels so as to predict mortality in patients with SIRS, sepsis, severe sepsis, and septic shock, we performed

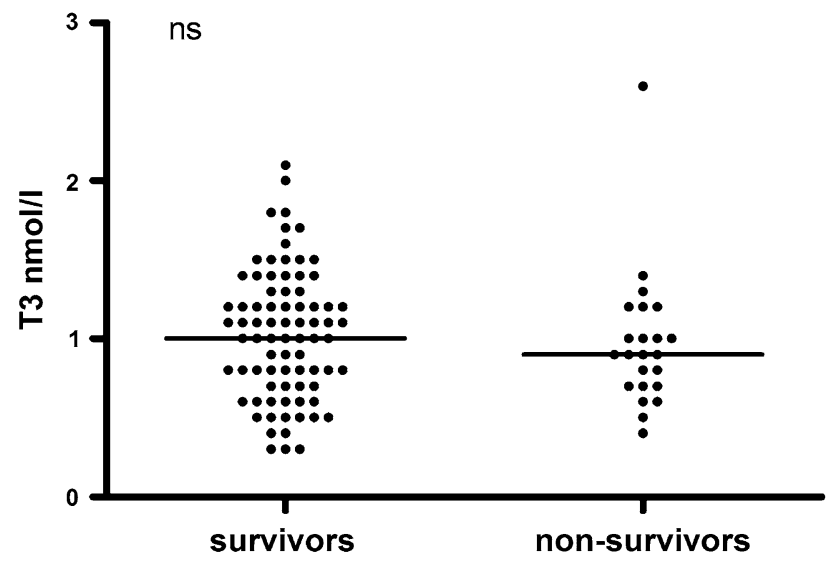

Fig. 3 T3 levels on admission in survivors and non-survivors Lines denote median values, each point represents one measurement. T3 triiodothyronine receiver operating characteristic (ROC) analysis. On comparison, the same ROC analysis values were obtained for CRP, PCT, the SAPS II, and APACHE II. The area under the curve (AUC) for T3 and fT4 on admission were 0.57 (IQR 0.47-0.67) and 0.53 (IQR 0.43-0.63), which was lower as compared to the AUC's of the SAPS II [AUC 0.76 (IQR 0.66-0.83)] and APACHE II [AUC 0.70 (IQR $0.60-0.79)]$. On day 2 the AUCs of T3 and fT4 were 0.63 (IQR 0.52-0.73) and 0.59 (IQR 0.49-0.7) and at discharge or death, respectively the AUCs were 0.65 (IQR 0.55-0.75) and 0.68 (IQR 0.57-0.77).

\section{Discussion}

This study has demonstrated two major findings. First, plasma T3 levels were lower in patients with sepsis as compared to patients with SIRS despite similar mortality rates. Second, circulating thyroid hormone levels measured on admission were not different in survivors and
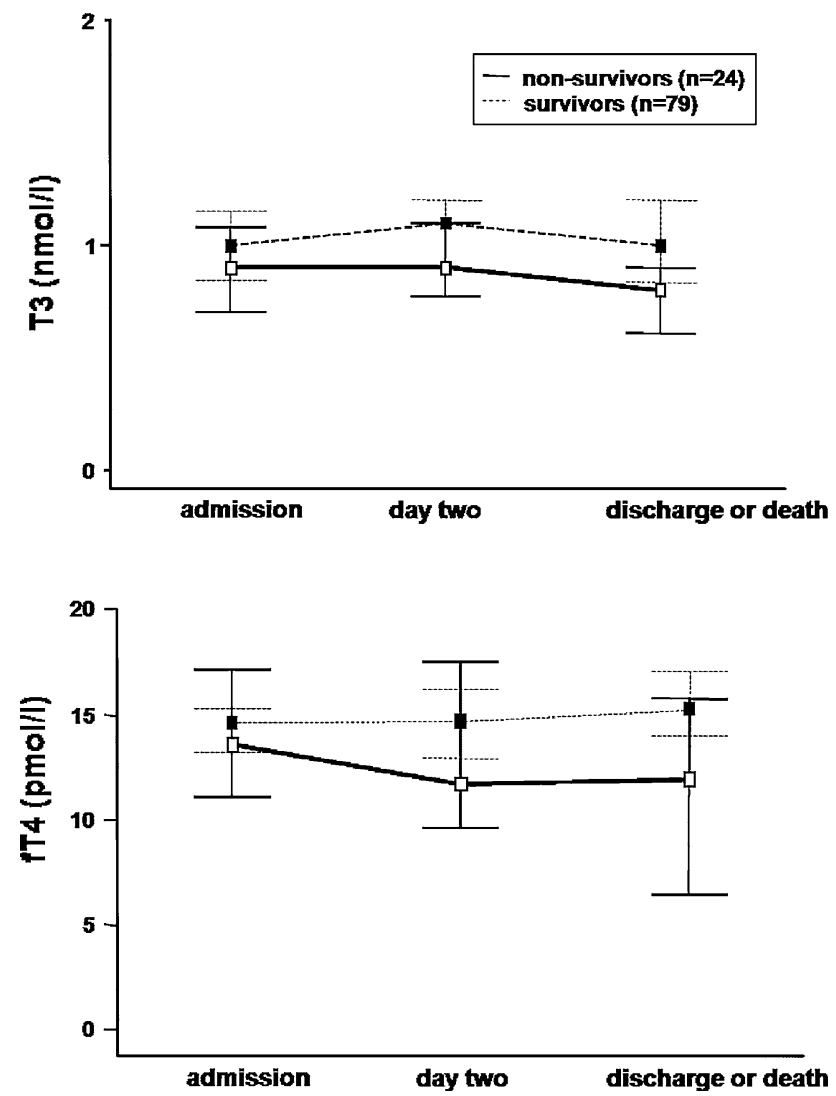

Fig. 4 T3 and fT4 levels during admission and follow-up in survivors and non-survivors. FT4 levels significantly decreased in non-survivors, while an increase in survivors was found $(P=0.003)$. On the day of death, non-survivors had lower T3 and fT4 levels as compared with survivors $(P=0.04$ and $P=0.02)$. Squares denote median values, whiskers indicate range. $T 3$ triiodothyronine, $f T 4$ free thyroxin 
non-survivors and thus, did not give helpful prognostic information.

The hypothesis that hormonal changes of the euthyroid sick syndrome predict outcome in critically ill patients remains unresolved [12, 13, 15, 16, 23-25]. Some studies showed a correlation of low T3 levels and low T4 levels with outcome in critically ill adults and children [13, 15, 23 , 25]. However, concurrent with our findings, a few studies did not detect any prognostic value of T3 and fT4 levels on admission to predict outcome [16, 17], and a few other found even relatively higher total T3 levels in nonsurviving compared with surviving children with meningococcal sepsis [12, 24].

Different theories have been propagated to explain the mechanism and significance of the euthyroid sick syndrome. Acute and sustained critical illness results in complex alterations in the thyroid axis in a bimodal pattern $[10,11]$. The acute initial phase is primarily characterized by changes in the peripheral metabolism and receptor binding of thyroid hormones, whereas in the chronic phase a diminished hypothalamic-pituitary-activity with a timely overlap of the two conditions is found $[10,11,26]$. The chronic phase of the euthyroid sick syndrome is characterized by reversibly diminished secretion of TSH which can be reactivated by thyrotropin-releasing hormone (TRH) administration [10, 11, 27].

The decrease in serum T3 and fT4 levels is a dynamic process, which develops over time [14, 28]. Hence, the typical adaptations of the thyroid axis may not be present in critically ill patients on admission to the ICU which is delayed with respect to the initiation of the critical illness per se. This might explain the lack of association between admission T3 and fT4 levels and outcome in our study $[12,23,24]$.

Accordingly, we found lower T3 and fT4 levels in nonsurvivors compared to survivors only during follow-up measurements. In agreement with this finding, Peeters et al. found T3 and T4 levels on day 5 to be significantly lower in critically ill patients who ultimately died compared to those who survived [14]. Similar to our study, those authors described an increase in peripheral thyroid hormones in surviving patients whereas thyroid hormones in patients who died decreased [14]. This is in line with previous studies suggesting that an increase in TSH drives the rise in T4 and thus points to the onset of recovery [29, 30]. There is evidence that a marked decrease in T4 levels is associated with a high probability of death [10, 31].

In addition, cytokines have a direct action on thyroid hormone synthesis through an effect on the hypothalamus and pituitary gland. It has been shown that shortterm infusion of IL6 to human volunteers causes a suppression of TSH but daily injections over 42 days caused only a modest decrease in T3 [32]. Antibody blockade of IL6, on the other hand, failed to induce the changes of the euthyroid sick syndrome in animal models [33]. Thus, IL6 could be involved in the euthyroid sick syndrome, but the potential interactions between cytokines and the hypothalamic pituitary thyroid axis complicate the pathophysiological understanding as cytokines themselves act in a network. However, the higher IL6 levels in patients with sepsis as compared with patients with SIRS in our study could explain the finding that septic patients had lower T3 levels compared to SIRS patients.

Furthermore, medication can alter thyroid hormones. Hence, dopamine given to many patients in an ICU plays a role in the development of euthyroid sick syndrome. Dopamine treatment depresses the thyroid axis per se and can aggravate the euthyroid sick syndrome by suppression of the TSH secretion as well as the TSH response to TRH [34-36]. 50\% of the patients who died during our study were treated with dopamine compared to only $36.7 \%$ of patients who were discharged. This could be another explanation for the lower T3 levels in non-survivors compared with survivors during follow-up.

The question whether critical ill patients with low thyroid hormones should be treated with thyroid hormone replacement is controversially disputed [27, 37-40]. In a small study, infusion of T4 to critically ill patients restored $\mathrm{T} 4$ levels but failed to show a clinical benefit [38]. In a study of severely burned patients given $200 \mu \mathrm{g}$ T3 daily, there was again no evidence of a beneficial or a disadvantageous effect [39]. However, improved myocardial function and the need for a reduced amount of vasopressor have been observed with T3 infusion in adults upon coronary artery bypass surgery [37, 40]. Pioneering studies by van den Berghe in patients with severe euthyroid sick syndrome showed that co-administration of TRH, growth hormone-releasing hormone, and growth hormone-releasing peptide 2 reduces protein catabolism by restoring physiologic hormone levels [27, 41]. The lack of an association of low T3/fT4 levels with the outcome in this study questions the potential usefulness of thyroid hormone treatment in critically ill patients as advocated by some authors [38, 39]. Only prospective intervention studies can give conclusive answers to this question.

Some limitations of this study merit consideration. First, this study is observational in nature. Second, the number of events (deaths) in this analysis is of only moderate size limiting the statistical power. Third, we analyzed patients with a diversity of conditions, but therewith reflecting the real-life situation on a medical ICU, which is dominated by heterogeneity. Fourth, we did not measure TSH levels which, most likely, would not have changed the main conclusion of our study. 


\section{Conclusion}

In conclusion, T3 and fT4 levels on admission were not prognostic in septic and non-septic critically ill patients. A decrease of fT4 levels during the follow-up may point to adverse outcome, whereas increasing peripheral thyroid hormones may be an indicator for a good prognostic course.

Acknowledgments The authors wish to thank the members of the laboratory of chemical pathology of the University Hospital Basel and the staff of the medical ICU, namely Rudolf Ritz and Hartmut Schächinger, for their support during the study.

Conflict of interest All authors declare that there is no conflict of interest.

\section{References}

1. D.C. Angus, R.S. Wax, Epidemiology of sepsis: an update. Crit. Care Med. 29, S109-S116 (2001)

2. G.S. Martin, D.M. Mannino, S. Eaton, M. Moss, The epidemiology of sepsis in the United States from 1979 through 2000. N. Engl. J. Med. 348, 1546-1554 (2003)

3. W.A. Knaus, E.A. Draper, D.P. Wagner, J.E. Zimmerman, APACHE II: a severity of disease classification system. Crit. Care Med. 13, 818-829 (1985)

4. J.R. Le Gall, S. Lemeshow, F. Saulnier, A new Simplified Acute Physiology Score (SAPS II) based on a European/North American multicenter study. JAMA 270, 2957-2963 (1993)

5. M. Christ-Crain, N.G. Morgenthaler, J. Struck, S. Harbarth, A. Bergmann, B. Muller, Mid-regional pro-adrenomedullin as a prognostic marker in sepsis: an observational study. Crit. Care 9, R816-R824 (2005)

6. F. de Groof, K.F. Joosten, J.A. Janssen, E.D. de Kleijn, J.A. Hazelzet, W.C. Hop, P. Uitterlinden, J. van Doorn, A.C. HokkenKoelega, Acute stress response in children with meningococcal sepsis: important differences in the growth hormone/insulin-like growth factor I axis between nonsurvivors and survivors. J. Clin. Endocrinol. Metab. 87, 3118-3124 (2002)

7. P. Schuetz, M. Christ-Crain, N.G. Morgenthaler, J. Struck, A. Bergmann, B. Muller, Circulating precursor levels of endothelin1 and adrenomedullin, two endothelium-derived, counteracting substances, in sepsis. Endothelium 14, 345-351 (2007)

8. M. Christ-Crain, D. Stolz, S. Jutla, O. Couppis, C. Muller, R. Bingisser, P. Schuetz, M. Tamm, R. Edwards, B. Muller, A.B. Grossman, Free and total cortisol levels as predictors of severity and outcome in community-acquired pneumonia. Am. J. Respir. Crit. Care Med. 176, 913-920 (2007)

9. R. Docter, E.P. Krenning, M. de Jong, G. Hennemann, The sick euthyroid syndrome: changes in thyroid hormone serum parameters and hormone metabolism. Clin. Endocrinol. (Oxf) 39, 499-518 (1993)

10. L.J. De Groot, Dangerous dogmas in medicine: the nonthyroidal illness syndrome. J. Clin. Endocrinol. Metab. 84, 151-164 (1999)

11. G. Van den Berghe, F. de Zegher, R. Bouillon, Clinical review 95: acute and prolonged critical illness as different neuroendocrine paradigms. J. Clin. Endocrinol. Metab. 83, 1827-1834 (1998)

12. K.F. Joosten, E.D. de Kleijn, M. Westerterp, M. de Hoog, F.C. Eijck, W.C.J. Hop, E.V. Voort, J.A. Hazelzet,
A.C. Hokken-Koelega, Endocrine and metabolic responses in children with meningoccocal sepsis: striking differences between survivors and nonsurvivors. J. Clin. Endocrinol. Metab. 85, 3746-3753 (2000)

13. P.M. Rothwell, P.G. Lawler, Prediction of outcome in intensive care patients using endocrine parameters. Crit. Care Med. 23, 78-83 (1995)

14. R.P. Peeters, P.J. Wouters, H. van Toor, E. Kaptein, T.J. Visser, G. Van den Berghe, Serum 3,3',5'-triiodothyronine (rT3) and 3,5,3'-triiodothyronine/rT3 are prognostic markers in critically ill patients and are associated with postmortem tissue deiodinase activities. J. Clin. Endocrinol. Metab. 90, 4559-4565 (2005)

15. K. Plikat, J. Langgartner, R. Buettner, L.C. Bollheimer, U. Woenckhaus, J. Scholmerich, C.E. Wrede, Frequency and outcome of patients with nonthyroidal illness syndrome in a medical intensive care unit. Metabolism 56, 239-244 (2007)

16. R. Lodha, S. Vivekanandhan, M. Sarthi, S. Arun, S.K. Kabra, Thyroid function in children with sepsis and septic shock. Acta Paediatr. 96, 406-409 (2007)

17. D.C. Ray, G.B. Drummond, E. Wilkinson, G.J. Beckett, Relationship of admission thyroid function tests to outcome in critical illness. Anaesthesia 50, 1022-1025 (1995)

18. B. Muller, K.L. Becker, H. Schachinger, P.R. Rickenbacher, P.R. Huber, W. Zimmerli, R. Ritz, Calcitonin precursors are reliable markers of sepsis in a medical intensive care unit. Crit. Care Med. 28, 977-983 (2000)

19. P. Schuetz, B. Muller, C. Nusbaumer, M. Wieland, M. ChristCrain, Circulating levels of GH predict mortality and complement prognostic scores in critically ill medical patients. Eur. J. Endocrinol. 160, 157-163 (2009)

20. 1992 American College of Chest Physicians/Society of Critical Care Medicine Consensus Conference: definitions for sepsis and organ failure and guidelines for the use of innovative therapies in sepsis. Crit. Care Med. 20:864-874

21. R.C. Bone, R.A. Balk, F.B. Cerra, R.P. Dellinger, A.M. Fein, W.A. Knaus, R.M. Schein, W.J. Sibbald, Definitions for sepsis and organ failure and guidelines for the use of innovative therapies in sepsis. The ACCP/SCCM consensus conference committee. American college of chest physicians/society of critical care medicine. Chest 101, 1644-1655 (1992)

22. G.C. Zucchelli, A. Pilo, M.R. Chiesa, S. Masini, Systematic differences between commercial immunoassays for free thyroxine and free triiodothyronine in an external quality assessment program. Clin. Chem. 40, 1956-1961 (1994)

23. M. den Brinker, B. Dumas, T.J. Visser, W.C. Hop, J.A. Hazelzet, D.A. Festen, A.C. Hokken-Koelega, K.F. Joosten, Thyroid function and outcome in children who survived meningococcal septic shock. Intensive Care Med. 31, 970-976 (2005)

24. M. den Brinker, K.F. Joosten, T.J. Visser, W.C. Hop, Y.B. de Rijke, J.A. Hazelzet, V.H. Boonstra, A.C. Hokken-Koelega, Euthyroid sick syndrome in meningococcal sepsis: the impact of peripheral thyroid hormone metabolism and binding proteins. J. Clin. Endocrinol. Metab. 90, 5613-5620 (2005)

25. M.F. Slag, J.E. Morley, M.K. Elson, T.W. Crowson, F.Q. Nuttall, R.B. Shafer, Hypothyroxinemia in critically ill patients as a predictor of high mortality. JAMA 245, 43-45 (1981)

26. R.P. Peeters, M.H. Kester, P.J. Wouters, E. Kaptein, H. van Toor, T.J. Visser, G. Van den Berghe, Increased thyroxine sulfate levels in critically ill patients as a result of a decreased hepatic type I deiodinase activity. J. Clin. Endocrinol. Metab. 90, 6460-6465 (2005)

27. G. Van den Berghe, F. de Zegher, R.C. Baxter, J.D. Veldhuis, P. Wouters, M. Schetz, C. Verwaest, E. Van der Vorst, P. Lauwers, R. Bouillon, C.Y. Bowers, Neuroendocrinology of prolonged critical illness: effects of exogenous thyrotropin-releasing hormone and its combination with growth hormone secretagogues. J. Clin. Endocrinol. Metab. 83, 309-319 (1998) 
28. A. Boelen, J. Kwakkel, D.C. Thijssen-Timmer, A. Alkemade, E. Fliers, W.M. Wiersinga, Simultaneous changes in central and peripheral components of the hypothalamus-pituitary-thyroid axis in lipopolysaccharide-induced acute illness in mice. J. Endocrinol. 182, 315-323 (2004)

29. V. Bacci, G.C. Schussler, T.B. Kaplan, The relationship between serum triiodothyronine and thyrotropin during systemic illness. J. Clin. Endocrinol. Metab. 54, 1229-1235 (1982)

30. P.S. Hamblin, S.A. Dyer, V.S. Mohr, B.A. Le Grand, C.F. Lim, D.V. Tuxen, D.J. Topliss, J.R. Stockigt, Relationship between thyrotropin and thyroxine changes during recovery from severe hypothyroxinemia of critical illness. J. Clin. Endocrinol. Metab. 62, 717-722 (1986)

31. L.S. Maldonado, G.H. Murata, J.M. Hershman, G.D. Braunstein, Do thyroid function tests independently predict survival in the critically ill? Thyroid 2, 119-123 (1992)

32. J.M. Stouthard, T. van der Poll, E. Endert, P.J. Bakker, C.H. Veenhof, H.P. Sauerwein, J.A. Romijn, Effects of acute and chronic interleukin-6 administration on thyroid hormone metabolism in humans. J. Clin. Endocrinol. Metab. 79, 1342-1346 (1994)

33. A. Boelen, M.C. Platvoet-ter Schiphorst, W.M. Wiersinga, Immunoneutralization of interleukin-1, tumor necrosis factor, interleukin- 6 or interferon does not prevent the LPS-induced sick euthyroid syndrome in mice. J. Endocrinol. 153, 115-122 (1997)

34. T. Schilling, M. Grundling, C.M. Strang, K.U. Moritz, W. Siegmund, T. Hachenberg, Effects of dopexamine, dobutamine or dopamine on prolactin and thyreotropin serum concentrations in high-risk surgical patients. Intensive Care Med. 30, 1127-1133 (2004)
35. G. Van den Berghe, F. de Zegher, Anterior pituitary function during critical illness and dopamine treatment. Crit. Care Med. 24, 1580-1590 (1996)

36. G. Van den Berghe, F. de Zegher, P. Lauwers, Dopamine and the sick euthyroid syndrome in critical illness. Clin. Endocrinol. (Oxf) 41, 731-737 (1994)

37. E. Bennett-Guerrero, J.L. Jimenez, W.D. White, E.B. D'Amico, B.I. Baldwin, D.A. Schwinn, Cardiovascular effects of intravenous triiodothyronine in patients undergoing coronary artery bypass graft surgery. A randomized, double-blind, placebo-controlled trial. Duke T3 study group. JAMA 275, 687-692 (1996)

38. G.A. Brent, J.M. Hershman, Thyroxine therapy in patients with severe nonthyroidal illnesses and low serum thyroxine concentration. J. Clin. Endocrinol. Metab. 63, 1-8 (1986)

39. R.A. Becker, G.M. Vaughan, M.G. Ziegler, L.G. Seraile, I.W. Goldfarb, E.H. Mansour, W.F. McManus, B.A. Pruitt Jr., A.D. Mason Jr., Hypermetabolic low triiodothyronine syndrome of burn injury. Crit. Care Med. 10, 870-875 (1982)

40. J.D. Klemperer, I. Klein, M. Gomez, R.E. Helm, K. Ojamaa, S.J. Thomas, O.W. Isom, K. Krieger, Thyroid hormone treatment after coronary-artery bypass surgery. N. Engl. J. Med. 333, 1522-1527 (1995)

41. G. Van den Berghe, R.C. Baxter, F. Weekers, P. Wouters, C.Y. Bowers, A. Iranmanesh, J.D. Veldhuis, R. Bouillon, The combined administration of GH-releasing peptide-2 (GHRP-2), TRH and $\mathrm{GnRH}$ to men with prolonged critical illness evokes superior endocrine and metabolic effects compared to treatment with GHRP-2 alone. Clin. Endocrinol. (Oxf) 56, 655-669 (2002) 